\title{
An Averaging Principle for Stochastic Differential Delay Equations with Fractional Brownian Motion
}

\author{
Yong Xu, Bin Pei, and Yongge Li \\ Department of Applied Mathematics, Northwestern Polytechnical University, Xian 710072, China \\ Correspondence should be addressed to Yong Xu; hsux3@nwpu.edu.cn
}

Received 15 November 2013; Accepted 19 December 2013; Published 22 January 2014

Academic Editor: Yaozhong Hu

Copyright (C) 2014 Yong Xu et al. This is an open access article distributed under the Creative Commons Attribution License, which permits unrestricted use, distribution, and reproduction in any medium, provided the original work is properly cited.

\begin{abstract}
An averaging principle for a class of stochastic differential delay equations (SDDEs) driven by fractional Brownian motion (fBm) with Hurst parameter in $(1 / 2,1)$ is considered, where stochastic integration is convolved as the path integrals. The solutions to the original SDDEs can be approximated by solutions to the corresponding averaged SDDEs in the sense of both convergence in mean square and in probability, respectively. Two examples are carried out to illustrate the proposed averaging principle.
\end{abstract}

\section{Introduction}

The averaging principle in stochastic dynamical systems is often used, and it is useful and effective for exploring stochastic differential equations (SDEs) in many different fields [1-4]. In Gaussian random fluctuations case, this analytic technique has been developed by Stratonovich [5, 6] and Khasminskii $[7,8]$. Shortly afterwards, researchers began to study the averaging principle of SDEs driven by Poisson noises [9, 10]. Recently, Zhu and his coworkers also investigated this averaging principle for a class of nonlinear systems with Poisson noises [11-13]. Instead of Poisson noises, $\mathrm{Xu}$ et al. established an averaging principle for SDEs with Lévy noise [14].

However, random fluctuations with long-range dependence, or correlated noises, are abundant, which may be modeled by fractional Brownian motion ( $\mathrm{fBm}$ ) with Hurst parameter in $(1 / 2,1)$. Therefore, SDEs with $\mathrm{fBm}$ have played an increasingly significant role in various fields of applications, such as hydrology, queueing theory, and mathematical finance [15-18]. Against this background, Xu et al. presented an averaging principle for SDEs with $\mathrm{fBm}$ [19]. It should be noted that one assumes the future states will have nothing to do with the past states besides the present states of the systems, for most of the applications. However, under closer scrutiny, it becomes apparent that a more realistic model would include some of the past states of the systems. Fortunately, stochastic differential delay equations (SDDEs) give a mathematical formulation for such kinds of systems. For this reason, SDDEs have attracted more and more attentions except the averaging principle for SDDEs driven by $\mathrm{fBm}[20-$ 22].

Therefore, we in the present paper will consider an averaging principle for the following SDDEs on $R^{d}$ of the form:

$$
\begin{aligned}
X(t)= & X(0)+\int_{0}^{t} b(s, X(s)) d s \\
& +\int_{0}^{t} \sigma(s, X(s-\tau)) d^{-} B^{H}(s), \quad t \in(0, T], \\
& X(t)=\eta(t), \quad t \in[-\tau, 0],
\end{aligned}
$$

where $\tau$ denotes a strictly positive time delay, $B^{H}=\left(B^{H, j}, j=\right.$ $1, \ldots m)$ are independent $\mathrm{fBm}$ with Hurst parameter $H$ in $(1 / 2,1)$ defined in a complete probability space $(\Omega, \mathscr{F}, P)$, and $b(s, X)$ the hereditary term, which is a measurable function, depends on the path $\left\{X\left(s_{1}\right),-\tau<s_{1}<s\right\}$, while $\eta[-\tau, 0] \rightarrow \mathbb{R}^{d}$ is a continuous and smooth function. $\sigma(s, X)$ is a measurable function. $\int_{0}^{t} \cdot d^{-} B^{H}(s)$ represents the forward integral which is interpreted in Definition 1. 
The simplified systems to (1) will be introduced as the so-called averaged systems, whose solutions will be applied to approximate the solutions of the original systems. And, we prove that the solutions of the original SDDEs converge to solutions of the corresponding averaged SDDEs in the sense of mean square and probability. In addition, the similar conclusion holds for SDDEs with $\mathrm{fBm}$, where the stochastic differential or stochastic integral is of symmetric and backward types.

\section{Some Preliminaries}

Since SDEs are interpreted via stochastic integrals, it is necessary to specify the integrals with respect to fBm [23-26].

Firstly, the process of $\mathrm{fBm}$ is Gaussian, and, hence we can develop a stochastic calculus of variation [16, 24, 26] (or Malliavin calculus) with respect to $\mathrm{fBm}$.

Let $g: \mathbb{R}_{+} \rightarrow \mathbb{R}$ be Borel measurable, $1 / 2<H<1$. $\varphi: \mathbb{R}_{+} \times \mathbb{R}_{+} \rightarrow \mathbb{R}_{+}$is given by

$$
\varphi(t, s)=H(2 H-1)|t-s|^{2 H-2}, \quad t, s \in \mathbb{R}_{+} .
$$

Then we say that $g \in L_{\varphi}^{2}\left(\mathbb{R}_{+}\right)$if

$$
\|g\|_{\varphi}^{2}=\int_{\mathbb{R}_{+}} \int_{\mathbb{R}_{+}} g(t) g(s) \varphi(t, s) d s d t<\infty
$$

If we equip $L_{\varphi}^{2}$ with the inner product

$$
\begin{array}{r}
\left\langle g_{1}, g_{2}\right\rangle_{\varphi}=\int_{\mathbb{R}_{+}} \int_{\mathbb{R}_{+}} g_{1}(t) g_{2}(s) \varphi(t, s) d s d t, \\
g_{1}, g_{2} \in L_{\varphi}^{2}\left(\mathbb{R}_{+}\right),
\end{array}
$$

then $L_{\varphi}^{2}\left(\mathbb{R}_{+}\right)$becomes a separable Hilbert space.

Let $\mathcal{S}$ be the set of smooth and cylindrical random variables of the form

$$
F=f\left(B^{H}\left(\psi_{1}\right), B^{H}\left(\psi_{2}\right) \cdots B^{H}\left(\psi_{n}\right)\right),
$$

where $n \geq 1, f \in \mathscr{C}_{b}^{\infty}\left(\mathbb{R}^{n}\right)$ (i.e., $f$ and all its partial derivatives are bounded), $\varphi_{i} \in \mathscr{H}, i=1,2 \ldots n$, and $\mathscr{H}$ is a Hilbert space. The elements of $\mathscr{H}$ may not be functions but distributions of negative order. Thanks to this reason, it is convenient to introduce the space $|\mathscr{H}|$ of measurable function $h$ on $[0, T]$ satisfying

$$
\|h\|_{|\mathscr{H}|}^{2}=\int_{0}^{T} \int_{0}^{T}|h(t)||h(s)| \varphi(t, s) d s d t<\infty .
$$

And it is not difficult to show that $|\mathscr{H}|$ is a Banach space with the norm $\|\cdot\|_{|\mathscr{H}|}^{2}$.

The derivative operator $D_{t}^{H}$ of a smooth and cylindrical random variable $F$ is defined as the $\mathscr{H}$-valued random variable:

$$
D_{t}^{H} F=\sum_{i=1}^{n} \frac{\partial f}{\partial x_{i}}\left(B^{H}\left(\psi_{1}\right), B^{H}\left(\psi_{2}\right) \cdots B^{H}\left(\psi_{n}\right)\right) \psi_{i} .
$$

Then, for any $p \geq 1$, the derivative operator $D_{t}^{H}$ is a closable operator from $L^{p}(\Omega)$ into $L^{p}(\Omega ; \mathscr{H})$. In addition, we denote $D_{t}^{H, k}$ as the iteration of the derivative operator for any integer $k \geq 1$. And the Sobolev space $\mathbb{D}^{k, p}$ is the closure of $\mathcal{S}$ with respect to the norm for any $p \geq 1(\otimes$ denotes the tensor product)

$$
\|F\|_{k, p}^{p}=E|F|^{p}+E \sum_{j=1}^{k}\left\|D_{t}^{H, j}\right\|_{\mathscr{H}^{\otimes j}}^{p} .
$$

Similarly, for a Hilbert space $U$, we denote by $\mathbb{D}^{k, p}(U)$ the corresponding Sobolev space of $U$-valued random variables. For any $p>1$ we denote by $\mathbb{D}^{1, p}(|\mathscr{H}|)$ the subspace of $\mathbb{D}^{1, p}(\mathscr{H})$ formed by the elements $h$ such that $h \in|\mathscr{H}|$.

By [23], we introduce the $\varphi$-derivative of $F$ :

$$
D_{t}^{\varphi} F=\int_{\mathbb{R}_{+}} \varphi(t, v) D_{v}^{H} F d v .
$$

Refer to $[23,26]$ to obtain more details.

Secondly, recall the definitions of the three types of pathwise integrals introduced by Russo and Vallois in [27].

Definition 1. Let $u(t)$ be a stochastic process with integrable trajectories.

(1) The symmetric integral $\int_{0}^{T} u(s) d \circ B^{H}(s)$ of $u(t)$ with respect to $B^{H}(t)$ is defined as the limit in probability as $\varepsilon$ tends to zero of

$$
\frac{1}{2 \varepsilon} \int_{0}^{T} u(s)\left[B^{H}(s+\varepsilon)-B^{H}(s-\varepsilon)\right] d s,
$$

provided this limit exists.

(2) The forward integral $\int_{0}^{T} u(s) d^{-} B^{H}(s)$ of $u(t)$ with respect to $B^{H}(t)$ is defined as the limit in probability as $\varepsilon$ tends to zero of

$$
\frac{1}{\varepsilon} \int_{0}^{T} u(s)\left[\frac{B^{H}(s+\varepsilon)-B^{H}(s)}{\varepsilon}\right] d s,
$$

provided this limit exists.

(3) The backward integral $\int_{0}^{T} u(s) d^{+} B^{H}(s)$ of $u(t)$ with respect to $B^{H}(t)$ is defined as the limit in probability as $\varepsilon$ tends to zero of

$$
\frac{1}{\varepsilon} \int_{0}^{T} u(s)\left[\frac{B^{H}(s-\varepsilon)-B^{H}(s)}{\varepsilon}\right] d s,
$$

provided this limit exists.

At last, we introduce some auxiliary results.

Lemma 2. Let $u(t)$ be a stochastic process in the space $\mathbb{D}^{1,2}(|\mathscr{H}|)$ and satisfy

$$
\int_{0}^{T} \int_{0}^{T}\left|D_{s}^{H} u(t)\right||t-s|^{2 H-2} d s d t<\infty
$$


By Remark 1 in [26] and Proposition 6.2.3 in [23], then the symmetric integral coincides with the forward and backward integrals.

Definition 3. The space $\mathscr{L}_{\varphi}[0, T]$ of integrands is defined as the family of stochastic processes $u(t)$ on $[0, T]$, if $E\|u(t)\|_{\varphi}^{2}<$ $\infty, u(t)$ is $\varphi$-differentiable, the trace of $D_{s}^{\varphi} u(t)$ exists, $0 \leq s \leq$ $T, 0 \leq t \leq T$, and

$$
E \int_{0}^{T} \int_{0}^{T}\left[D_{s}^{\varphi} u(t)\right]^{2} d s d t<\infty
$$

and for each sequence of partitions $\left(\pi_{n}, n \in \mathbb{N}\right)$ such that $\left|\pi_{n}\right| \rightarrow 0$ as $n \rightarrow \infty$,

$$
\begin{gathered}
\sum_{i=0}^{n-1} E\left[\int_{t_{i}^{(n)}}^{t_{i+1}^{(n)}} \int_{t_{j}^{(n)}}^{t_{j+1}^{(n)}}\left|D_{s}^{\varphi} u_{t_{i}^{(n)}}^{\pi} D_{t}^{\varphi} u_{t_{j}^{(n)}}^{\pi}-D_{s}^{\varphi} u_{t} D_{t}^{\varphi} u_{s}\right| d s d t\right], \\
E\left[\left\|u^{\pi}-u\right\|_{\varphi}^{2}\right]
\end{gathered}
$$

tend to 0 as $n \rightarrow \infty$, where $\pi_{n}=t_{0}^{(n)}<t_{1}^{(n)}<\cdots<t_{n-1}^{(n)}<$ $t_{n}^{(n)}=T,|\pi|:=\max _{i}\left(t_{i+1}-t_{i}\right)$ and $u^{\pi}=u_{t_{i}}$.

Remark 4. If $u(t) \in \mathscr{L}_{\varphi}[0, T]$, then the three types of pathwise integrals exist and the following relations hold:

$$
\begin{aligned}
& \int_{0}^{T} u(s) d \circ B^{H}(s)=\int_{0}^{T} u(s) d \diamond B^{H}(s)+\int_{0}^{T} D_{s}^{\varphi} u(s) d s, \\
& \int_{0}^{T} u(s) d^{-} B^{H}(s)=\int_{0}^{T} u(s) \diamond d B^{H}(s)+\int_{0}^{T} D_{s}^{\varphi} u(s) d s, \\
& \int_{0}^{T} u(s) d^{+} B^{H}(s)=\int_{0}^{T} u(s) \diamond d B^{H}(s)+\int_{0}^{T} D_{s}^{\varphi} u(s) d s,
\end{aligned}
$$

where $\diamond$ denotes the Wick product.

We note that the result of Remark 4 also follows by Proposition 6.2.2 in [23] and Lemma 2. The proof of Remark 4 can be found by Theorem 6.2.5 in [23].

Lemma 5. Let $u(s)$ be a stochastic process in $\mathscr{L}_{\varphi}[0, T]$; there exists a constant $C$ such that

$$
E\left[\int_{0}^{T} u(s) \diamond d B^{H}(s)\right]^{2} \leq H T^{2 H-1} E\left[\int_{0}^{T}|u(s)|^{2} d s\right]+C T^{2} .
$$

Lemma 6. Suppose that $u(s)$ is a stochastic process in $\mathscr{L}_{\varphi}[0, T]$; there exists a constant $C$ such that

$$
\begin{aligned}
& E\left[\int_{0}^{T} u(s) d \circ B^{H}(s)\right]^{2} \\
& \quad \leq 2 H T^{2 H-1} E\left[\int_{0}^{T}|u(s)|^{2} d s\right]+4 C T^{2} .
\end{aligned}
$$

The detailed proofs of Lemmas 5 and 6 are in [19].
Remark 7. In the same conditions with Lemmas 2, 5 and 6 and under Remark 4, we can get the symmetric, forward and backward integrals cases have the same conclusions as symmetric integral case in Lemma 6.

\section{An Averaging Principle for SDDEs with $\mathbf{f B m}$}

3.1. SDDEs Driven by fBm. One can define the integral with respect to $\mathrm{fBm}$ using a pathwise approach with $1 / 2<H<1$. Indeed, if there is a stochastic process $u(t)$, whose trajectories are $\lambda$-Hölder continuous with $\lambda>1-H$, then the RiemannStieltjes integral $\int_{0}^{T} u(s) d B^{H}(s)$ exists for each trajectory [28].

Moreover, the pathwise Riemann-Stieltjes integral coincides with the symmetric integral in the Russo-Vallois sense which has been proved in [25, 29]. Therefore, in terms of Lemma 2 and [29], we can conclude that the pathwise Riemann-Stieltjes integral coincides with the three types of pathwise integrals in this paper.

In this section the forward integral of SDDEs with respect to $\mathrm{fBm}$ is firstly concerned. Then, the other two kinds of cases are given subsequently.

Now, considering the following assumptions on the coefficients of (1),

(H1) $\sigma:[0, T] \times \mathbb{R}^{d} \rightarrow \mathbb{R}^{d} \times \mathbb{R}^{m}$ is a measurable function $\sigma(t, x)$ which is differentiable in $x$, and there exist some constants $0<\gamma, \delta \leq 1$ and for every $N \geq 0$ there exists $M_{N}>0$ such that the following properties hold:

(C1) $|\sigma(t, x)-\sigma(t, y)| \leq M_{0}|x-y|$, for all $x, y \in \mathbb{R}^{d}$, for all $t \in[0, T]$,

(C2) $\left|\partial x_{i} \sigma(t, x)-\partial y_{i} \sigma(t, y)\right| \leq M_{N}|x-y|^{\delta}$, for all $|x|,|y| \leq N$, for all $t \in[0, T]$, for each $i=$ $1, \ldots d$

(C3) $|\sigma(t, x)-\sigma(s, x)|+\left|\partial x_{i} \sigma(t, x)-\partial x_{i} \sigma(s, x)\right| \leq$ $M_{0}|t-s|^{\gamma}$, for all $x \in \mathbb{R}^{d}$, for all $t, s \in[0, T]$ for each $i=1, \ldots d$;

$(\mathrm{H} 2) b:[0, T] \times C\left(-\tau, T ; \mathbb{R}^{d}\right) \rightarrow \mathbb{R}^{d}$ is a measurable function such that, for every $t>0$ and $h \in$ $C\left(-\tau, T ; \mathbb{R}^{d}\right), b(t, h)$ depends only on $\{h(s) ;-\tau<s<$ $t\}$.

Moreover, there exists $b_{0} \in L^{\rho}\left(0, T ; \mathbb{R}^{d}\right)$ with $\rho \geq 2$ and for all $N \geq 0$ there exists $L_{N}>0$ such that

(C4) $|b(t, x)-b(t, y)| \leq L_{N} \sup _{-\tau \leq s \leq t}|x(s)-y(s)|$, for all $t \in[0, T]$, for all $x, y,\|x\|_{\infty(\tau)} \leq N,\|y\|_{\infty(\tau)} \leq$ $N$, where $\|x\|_{\infty(\tau)}=\sup _{s \in[-\tau, T]}|x(s)|,\|y\|_{\infty(\tau)}=$ $\sup _{s \in[-\tau, T]}|y(s)|$,

(C5) $|b(t, x)| \leq L_{0} \sup _{-\tau \leq s \leq t}|x(s)|+b_{0}(t)$, for all $t \in$ $[0, T]$.

The existence and uniqueness of solutions of the SDDEs driven by $\mathrm{fBm}$ under the above assumptions ( $\mathrm{H} 1-\mathrm{H} 2)$ have been proved by Ferrante and Rovira in [30]. 
3.2. Main Results. In this section, we discuss a standard SDDE using an averaging principle.

The standard SDDE is defined as

$$
\begin{aligned}
X_{\varepsilon}(t)= & X(0)+\varepsilon^{2 H} \int_{0}^{t} b\left(s, X_{\varepsilon}(s)\right) d s \\
& +\varepsilon^{H} \int_{0}^{t} \sigma\left(s, X_{\varepsilon}(s-\tau)\right) d^{-} B^{H}(s),
\end{aligned}
$$

where $X(0)=\eta(0)$ is a given $d$-dimensional random variable as the initial condition, $t \in[0, T]$ and the coefficients have the same conditions as in (1), and $\varepsilon \in\left(0, \varepsilon_{0}\right]$ is a positive parameter with $\varepsilon_{0}$ a fixed number.

Then, we introduce the averaged SDDE:

$$
\begin{aligned}
Z_{\varepsilon}(t)= & X(0)+\varepsilon^{2 H} \int_{0}^{t} \bar{b}\left(Z_{\varepsilon}(s)\right) d s \\
& +\varepsilon^{H} \int_{0}^{t} \bar{\sigma}\left(Z_{\varepsilon}(s-\tau)\right) d^{-} B^{H}(s) .
\end{aligned}
$$

Assume that (C1)-(C5) are satisfied for (20); besides the mappings $\bar{\sigma}: \mathbb{R}^{d} \rightarrow \mathbb{R}^{d} \times \mathbb{R}^{m}, \bar{b}: C\left(-\tau, T ; \mathbb{R}^{d}\right) \rightarrow \mathbb{R}^{d}$ are measurable.

Moreover, we presume the coefficients meet the following additional inequalities:

$$
\begin{aligned}
& \text { (C6) }\left(1 / T_{1}\right) \int_{0}^{T_{1}}|b(s, y)-\bar{b}(y)| d s \leq \varphi_{1}\left(T_{1}\right)(1+|y|), \\
& \text { (C7) }\left(1 / T_{1}\right) \int_{0}^{T_{1}}|\sigma(s, y)-\bar{\sigma}(y)|^{2} d s \leq \varphi_{2}\left(T_{1}\right)\left(1+|y|^{2}\right),
\end{aligned}
$$

where $T_{1} \in[0, T], \varphi_{i}\left(T_{1}\right)$ are positive bounded functions with $\lim _{T_{1} \rightarrow \infty} \varphi_{i}\left(T_{1}\right)=0, i=1,2$.

Obviously, under similar conditions such as (19), (20) also has a unique solution $Z_{\varepsilon}(t)$. In the rest of the paper, we will consider the connections between the solution processes $Z_{\varepsilon}(t)$ and $X_{\varepsilon}(t)$.

Now we prove the following main theorems to show relationship between solution processes $Z_{\varepsilon}(t)$ and $X_{\varepsilon}(t)$.

Theorem 8. Assume that the original SDDE (19) and the averaged SDDE (20) both satisfy the assumptions (H1), (H2) and (C6), (C7). For a given arbitrarily small number $\delta_{1}>0$, there exist $L>0, \varepsilon_{1} \in\left(0, \varepsilon_{0}\right]$, and $\beta \in(0,1)$, such that, for any $\varepsilon \in\left(0, \varepsilon_{1}\right]$

$$
E\left(\left|X_{\varepsilon}(t)-Z_{\varepsilon}(t)\right|^{2}\right) \leq \delta_{1} .
$$

Proof. By the above analysis, we start with

$$
\begin{aligned}
X_{\varepsilon}(t)- & Z_{\varepsilon}(t) \\
= & \varepsilon^{2 H} \int_{0}^{t}\left[b\left(s, X_{\varepsilon}(s)\right)-\bar{b}\left(Z_{\varepsilon}(s)\right)\right] d s \\
& +\varepsilon^{H} \int_{0}^{t}\left[\sigma\left(s, X_{\varepsilon}(s-\tau)\right)-\bar{\sigma}\left(Z_{\varepsilon}(s-\tau)\right)\right] d^{-} B^{H}(s)
\end{aligned}
$$

and employ the following inequality for $m \in \mathbb{N}$ and $x_{1}$, $x_{2}, \ldots x_{m} \in \mathbb{R}$ :

$$
\left|x_{1}+x_{2}+\cdots x_{m}\right|^{2} \leq m\left(\left|x_{1}\right|^{2}+\left|x_{2}\right|^{2}+\cdots\left|x_{m}\right|^{2}\right) .
$$

We obtain that

$$
\begin{aligned}
& \left|X_{\varepsilon}(t)-Z_{\varepsilon}(t)\right|^{2} \\
& \leq 2 \varepsilon^{4 H}\left|\int_{0}^{t}\left[b\left(s, X_{\varepsilon}(s)\right)-\bar{b}\left(Z_{\varepsilon}(s)\right)\right] d s\right|^{2} \\
& \quad+2 \varepsilon^{2 H}\left|\int_{0}^{t}\left[\sigma\left(s, X_{\varepsilon}(s-\tau)\right)-\bar{\sigma}\left(Z_{\varepsilon}(s-\tau)\right)\right] d^{-} B^{H}(s)\right|^{2} \\
& =I_{1}^{2}+I_{2}^{2},
\end{aligned}
$$

where $[0, t] \subseteq[0, u] \subseteq[0, T], I_{i}, i=1,2$ denote the above terms, respectively.

Now we present some estimates for $I_{i}, i=1,2$.

Firstly, we apply the inequality (23) to yield

$$
\begin{aligned}
I_{1}^{2}= & 2 \varepsilon^{4 H}\left|\int_{0}^{t}\left[b\left(s, X_{\varepsilon}(s)\right)-\bar{b}\left(Z_{\varepsilon}(s)\right)\right] d s\right|^{2} \\
= & 2 \varepsilon^{4 H} \mid \int_{0}^{t}\left[b\left(s, X_{\varepsilon}(s)\right)-b\left(s, Z_{\varepsilon}(s)\right)\right. \\
& \left.+b\left(s, Z_{\varepsilon}(s)\right)-\bar{b}\left(Z_{\varepsilon}(s)\right)\right]\left.d s\right|^{2} \\
\leq & 4 \varepsilon^{4 H}\left|\int_{0}^{t}\left[b\left(s, X_{\varepsilon}(s)\right)-b\left(s, Z_{\varepsilon}(s)\right)\right] d s\right|^{2} \\
& +4 \varepsilon^{4 H}\left|\int_{0}^{t}\left[b\left(s, Z_{\varepsilon}(s)\right)-\bar{b}\left(Z_{\varepsilon}(s)\right)\right] d s\right|^{2} \\
= & I_{11}^{2}+I_{12}^{2},
\end{aligned}
$$

where

$$
\begin{gathered}
I_{11}^{2}=4 \varepsilon^{4 H}\left|\int_{0}^{t}\left[b\left(s, X_{\varepsilon}(s)\right)-b\left(s, Z_{\varepsilon}(s)\right)\right] d s\right|^{2}, \\
I_{12}^{2}=4 \varepsilon^{4 H}\left|\int_{0}^{t}\left[b\left(s, Z_{\varepsilon}(s)\right)-\bar{b}\left(Z_{\varepsilon}(s)\right)\right] d s\right|^{2} .
\end{gathered}
$$

By the Cauchy-Schwarz inequality for $I_{11}^{2}$, we arrive at

$$
I_{11}^{2} \leq 4 \varepsilon^{4 H} t \int_{0}^{t}\left|b\left(s, X_{\varepsilon}(s)\right)-b\left(s, Z_{\varepsilon}(s)\right)\right|^{2} d s .
$$

According to condition (C5), the elementary inequality $(a-b)^{2} \leq 2\left(a^{2}+b^{2}\right)$ and $b_{0} \in L^{\rho}\left(0, T ; \mathbb{R}^{d}\right)$, and taking expectation, we have

$$
\begin{aligned}
E\left|I_{11}\right|^{2} & =4 \varepsilon^{4 H} E\left|\int_{0}^{t}\left[b\left(s, X_{\varepsilon}(s)\right)-b\left(s, Z_{\varepsilon}(s)\right)\right] d s\right|^{2} \\
& \leq 4 \varepsilon^{4 H} t E \int_{0}^{t}\left[b\left(s, X_{\varepsilon}(s)\right)-b\left(s, Z_{\varepsilon}(s)\right)\right]^{2} d s \\
& \leq 8 \varepsilon^{4 H} t E \int_{0}^{t}\left[\left|b\left(s, X_{\varepsilon}(s)\right)\right|^{2}+\left|b\left(s, Z_{\varepsilon}(s)\right)\right|^{2}\right] d s
\end{aligned}
$$


Abstract and Applied Analysis

5

$$
\begin{aligned}
& \leq 8 \varepsilon^{4 H} u E \int_{0}^{u}\left\{\left[L_{0} \sup _{-\tau \leq s_{1} \leq s}\left|X_{\varepsilon}\left(s_{1}\right)\right|+b_{0}(s)\right]^{2}\right. \\
& \left.+\left[L_{0} \sup _{-\tau \leq s_{1} \leq s}\left|Z_{\varepsilon}\left(s_{1}\right)\right|+b_{0}(s)\right]^{2}\right\} d s \\
& \leq 8 \varepsilon^{4 H} u K_{11},
\end{aligned}
$$

where $K_{11}$ denotes constant.

Then, for $\left|I_{12}\right|^{2}$, using condition (C6), $\varphi_{1}\left(T_{1}\right)$ is a positive bounded function and taking expectation to yield

$$
\begin{aligned}
E\left|I_{12}\right|^{2} & \leq 4 \varepsilon^{4 H} t^{2} E\left[\frac{1}{t} \int_{0}^{t}\left|b\left(s, Z_{\varepsilon}(s)\right)-\bar{b}\left(Z_{\varepsilon}(s)\right)\right| d s\right]^{2} \\
& \leq 4 \varepsilon^{4 H} u^{2}\left(\sup _{0 \leq t \leq u} \varphi_{1}^{2}(t)\right)\left(1+E\left(\sup _{0 \leq t \leq u}\left|Z_{\varepsilon}(t)\right|^{2}\right)\right) \\
& \leq 4 \varepsilon^{4 H} u^{2} K_{12}
\end{aligned}
$$

where $K_{12}$ denotes constant.

Putting $\left|I_{11}\right|^{2}$ and $\left|I_{12}\right|^{2}$ together, we reach

$$
E\left|I_{1}\right|^{2}=E\left|I_{11}\right|^{2}+E\left|I_{12}\right|^{2} \leq 8 \varepsilon^{4 H} u K_{11}+4 \varepsilon^{4 H} u^{2} K_{12} .
$$

Now, taking expectation on $I_{2}^{2}$ we obtain

$$
\begin{gathered}
E\left|I_{2}\right|^{2} \\
=2 \varepsilon^{2 H} E\left|\int_{0}^{t}\left[\sigma\left(s, X_{\varepsilon}(s-\tau)\right)-\bar{\sigma}\left(Z_{\varepsilon}(s-\tau)\right)\right] d^{-} B^{H}(s)\right|^{2} \\
\leq 4 \varepsilon^{2 H} E \mid \int_{0}^{t}\left[\sigma\left(s, X_{\varepsilon}(s-\tau)\right)\right. \\
\left.+-\sigma\left(s, Z_{\varepsilon}(s-\tau)\right)\right]\left.d^{-} B^{H}(s)\right|^{2} \\
\left.-\bar{\sigma}\left(\varepsilon_{\varepsilon}(s-\tau)\right)\right]\left.d^{-} B^{H}(s)\right|^{2}=I_{21}^{2}+I_{22}^{2}
\end{gathered}
$$

where

$$
\begin{aligned}
I_{21}^{2}= & 4 \varepsilon^{2 H} \\
& \times E\left|\int_{0}^{t}\left[\sigma\left(s, X_{\varepsilon}(s-\tau)\right)-\sigma\left(s, Z_{\varepsilon}(s-\tau)\right)\right] d^{-} B^{H}(s)\right|^{2}, \\
I_{22}^{2}= & 4 \varepsilon^{2 H} E\left|\int_{0}^{t}\left[\sigma\left(s, Z_{\varepsilon}(s-\tau)\right)-\bar{\sigma}\left(Z_{\varepsilon}(s-\tau)\right)\right] d^{-} B^{H}(s)\right|^{2} .
\end{aligned}
$$

By Remark 7, Lemma 6, and (C1), we obtain

$$
\begin{aligned}
I_{21}^{2}= & 4 \varepsilon^{2 H} E \mid \int_{0}^{t}\left[\sigma\left(s, X_{\varepsilon}(s-\tau)\right)-\sigma\left(s, Z_{\varepsilon}(s-\tau)\right)\right] \\
& \times\left. d^{-} B^{H}(s)\right|^{2} \\
\leq & 4 \varepsilon^{2 H}\left\{2 H t ^ { 2 H - 1 } E \int _ { 0 } ^ { t } \left[\sigma\left(s, X_{\varepsilon}(s-\tau)\right)\right.\right. \\
\leq & \left.\left.4 \varepsilon^{2 H} M_{0}^{2}-\sigma\left(s, Z_{\varepsilon}(s-\tau)\right)\right]^{2} d s+4 C u^{2}\right\} \\
& \times\left(2 H t^{2 H-1} E\left[\int_{0}^{t}\left|X_{\varepsilon}(s-\tau)-Z_{\varepsilon}(s-\tau)\right|^{2} d s\right]\right. \\
& +8 \varepsilon^{2 H} u^{2 H-1} K_{211} E \int_{-\tau}^{0}\left|X_{\varepsilon}(s)-Z_{\varepsilon}(s)\right|^{2} d s \\
& +16 \varepsilon^{2 H} K_{212}, \\
\leq & 8 \varepsilon^{2 H} u^{2 H} u^{2 H-1} K_{211} \\
& +16 \varepsilon^{2 H} K_{212}^{u} K_{211} \int_{0}^{u} E\left|X_{\varepsilon}(s)-Z_{\varepsilon}(s)\right|^{2} d s \\
& \times\left(E \int_{0}^{2}\left|X_{\varepsilon}(s)-Z_{\varepsilon}(s)\right|^{2} d s\right. \\
& \left.+E \int_{\varepsilon}^{0}(s)-\left.Z_{\varepsilon}(s)\right|^{2} d s\right)
\end{aligned}
$$

where $K_{211}, K_{212}$ denote constants.

Due to condition (C7), we have

$$
\begin{aligned}
I_{22}^{2} & =4 \varepsilon^{2 H} E\left|\int_{0}^{t}\left[\sigma\left(s, Z_{\varepsilon}(s-\tau)\right)-\bar{\sigma}\left(Z_{\varepsilon}(s-\tau)\right)\right] d^{-} B^{H}(s)\right|^{2} \\
& \leq 4 \varepsilon^{2 H}\left\{2 H u ^ { 2 H - 1 } E \left[\int_{0}^{t} \mid \sigma\left(s, Z_{\varepsilon}(s-\tau)\right)\right.\right.
\end{aligned}
$$

$$
\left.\left.-\left.\bar{\sigma}\left(Z_{\varepsilon}(s-\tau)\right)\right|^{2} d s\right]+4 C u^{2}\right\}
$$

$\leq 8 \varepsilon^{2 H} H u^{2 H-1} t$

$$
\times E\left\{\frac{1}{t} \int_{0}^{t}\left|\sigma\left(s, Z_{\varepsilon}(s-\tau)\right)-\bar{\sigma}\left(Z_{\varepsilon}(s-\tau)\right)\right|^{2} d s\right\}
$$

$+16 \varepsilon^{2 H} u^{2} K_{222}$ 


$$
\begin{aligned}
\leq & 8 \varepsilon^{2 H} H u^{2 H}\left(\sup _{0 \leq t \leq u} \varphi_{2}(t)\right) \\
& \times\left[1+E\left(\sup _{-\tau \leq t \leq 0}\left|Z_{\varepsilon}(t)\right|^{2}\right)+E\left(\sup _{0 \leq t \leq u}\left|Z_{\varepsilon}(t)\right|^{2}\right)\right] \\
& +16 \varepsilon^{2 H} u^{2} K_{222} \\
\leq & 8 \varepsilon^{2 H} H u^{2 H} K_{221}+16 \varepsilon^{2 H} u^{2} K_{222} .
\end{aligned}
$$

Then

$$
\begin{aligned}
E\left|I_{2}\right|^{2} \leq & 8 \varepsilon^{2 H} u^{2 H-1} K_{211} \int_{0}^{u} E\left|X_{\varepsilon}(s)-Z_{\varepsilon}(s)\right|^{2} d s \\
& +8 \varepsilon^{2 H}\left(u^{2 H-1} K_{211}+K_{212}+H u^{2 H} K_{221}+u^{2} K_{222}\right) .
\end{aligned}
$$
get

Therefore, from the above discussions (30) and (35), we

$$
\begin{gathered}
E\left|X_{\varepsilon}(t)-Z_{\varepsilon}(t)\right|^{2} \\
\leq 8 \varepsilon^{2 H} u^{2 H-1} K_{211} \int_{0}^{u} E\left|X_{\varepsilon}(s)-Z_{\varepsilon}(s)\right|^{2} d s+8 \varepsilon^{4 H} u K_{11} \\
\quad+4 \varepsilon^{4 H} u^{2} K_{12}+8 \varepsilon^{2 H}\left(u^{2 H-1} K_{211}+K_{212}\right. \\
\left.+H u^{2 H} K_{221}+u^{2} K_{222}\right) .
\end{gathered}
$$

Now by the Gronwall-Bellman inequality, we obtain

$$
\begin{aligned}
& E\left|X_{\varepsilon}(t)-Z_{\varepsilon}(t)\right|^{2} \\
& \leq 4 \varepsilon^{2 H}\left[\varepsilon^{2}\left(2 u K_{11}+u^{2} K_{12}\right)+u^{2 H-1} K_{211}+K_{212}\right. \\
&\left.+H u^{2 H} K_{221}+u^{2} K_{222}\right] \exp \left(8 \varepsilon^{2 H} u^{2 H} K_{211}\right) .
\end{aligned}
$$

Select $\beta \in(0,1), L>0$, such that, for all $t \in\left(0, L \varepsilon^{-H \beta}\right) \subseteq$ $[0, T]$, we have

$$
E\left|X_{\varepsilon}(t)-Z_{\varepsilon}(t)\right|^{2} \leq K_{3} \varepsilon^{1-H \beta},
$$

where

$$
\begin{aligned}
K_{3}=[ & 8 L \varepsilon^{2 H+1} K_{11}+4 L^{2} \varepsilon^{2 H+1-H \beta} K_{12} \\
& +4 L^{2 H-1} \varepsilon^{2 H-1+2 H \beta-2 H^{2} \beta} K_{211}+4 \varepsilon^{2 H-1+H \beta} K_{212} \\
& \left.+4 H L^{2 H} \varepsilon^{2 H-1+H \beta-2 H^{2} \beta} K_{221}+4 L^{2} \varepsilon^{2 H-1-H \beta} K_{222}\right] \\
& \times \exp \left(8 \varepsilon^{2 H-2 H^{2} \beta} L^{2 H} K_{211}\right)
\end{aligned}
$$

is a constant.
Consequently, given any number $\delta_{1}>0$, we can select $\varepsilon_{1} \in\left(0, \varepsilon_{0}\right]$, such that, for every $\varepsilon \in\left(0, \varepsilon_{1}\right]$ and for $t \in$ $\left(0, L \varepsilon^{-H \beta}\right) \subseteq[0, T]$,

$$
E\left(\left|X_{\varepsilon}(t)-Z_{\varepsilon}(t)\right|^{2}\right) \leq \delta_{1} .
$$

This completes the proof.

Theorem 9. Assume that the original SDDE (19) and the averaged SDDE (20) both satisfy the assumptions (H1), (H2) and (C6), (C7). Then for any number $\delta_{2}>0$, there exist $L>0$ and $\beta \in(0,1)$, such that

$$
\lim _{\varepsilon \rightarrow 0} P\left(\left|X_{\varepsilon}(t)-Z_{\varepsilon}(t)\right|^{2}>\delta_{2}\right)=0 .
$$

Proof. On the basis of Theorem 8 and the Chebyshev-Markov inequality, for any given number $\delta_{2}>0$, one can have

$$
\begin{aligned}
P\left(\left|X_{\varepsilon}(t)-Z_{\varepsilon}(t)\right|^{2}>\delta_{2}\right) & \leq \frac{1}{\delta_{2}^{2}} E\left|X_{\varepsilon}(t)-Z_{\varepsilon}(t)\right|^{2} \\
& \leq \frac{K_{3} \varepsilon^{1-H \beta}}{\delta_{2}^{2}} .
\end{aligned}
$$

Let $\varepsilon \rightarrow 0$ and the required result follows.

This completes the proof.

Then we also can study the symmetric integral and backward integral of SDDEs with $\mathrm{fBm}$. On the basis of (19) and (20), we can get the original SDDEs and the averaged SDDEs

$$
\begin{aligned}
X_{\varepsilon}(t)= & X(0)+\varepsilon^{2 H} \int_{0}^{t} b\left(s, X_{\varepsilon}(s)\right) d s \\
& +\varepsilon^{H} \int_{0}^{t} \sigma\left(s, X_{\varepsilon}(s-\tau)\right) d \circ B^{H}(s), \\
Z_{\varepsilon}(t)= & X(0)+\varepsilon^{2 H} \int_{0}^{t} \bar{b}\left(Z_{\varepsilon}(s)\right) d s \\
& +\varepsilon^{H} \int_{0}^{t} \bar{\sigma}\left(Z_{\varepsilon}(s-\tau)\right) d \circ B^{H}(s), \\
X_{\varepsilon}(t)= & X(0)+\varepsilon^{2 H} \int_{0}^{t} b\left(s, X_{\varepsilon}(s)\right) d s \\
& +\varepsilon^{H} \int_{0}^{t} \sigma\left(s, X_{\varepsilon}(s-\tau)\right) d^{+} B^{H}(s), \\
& +\varepsilon^{H} \int_{0}^{t} \bar{\sigma}\left(Z_{\varepsilon}(s-\tau)\right) d^{+} B^{H}(s), \\
Z_{\varepsilon}(t)= & X(0)+\varepsilon^{2 H} \int_{0}^{t} \bar{b}\left(Z_{\varepsilon}(s)\right) d s
\end{aligned}
$$

where $X(0)=\eta(0)$ is the initial condition, and the coefficients satisfy the $(\mathrm{C} 1)-(\mathrm{C} 5)$.

Theorem 10. Assume that the original SDDEs (43a), (44a) and the averaged SDDEs (43b), (44b) both satisfy the assumptions 
(H1), (H2) and (C6), (C7). For a given arbitrarily small number $\delta_{3}>0$, there exist $\varepsilon_{2} \in\left(0, \varepsilon_{0}\right]$ and $\varepsilon \in\left(0, \varepsilon_{2}\right]$ :

$$
E\left(\left|X_{\varepsilon}(t)-Z_{\varepsilon}(t)\right|^{2}\right) \leq \delta_{3} .
$$

And then for any number $\delta_{4}>0$, we can get

$$
\lim _{\varepsilon \rightarrow 0} P\left(\left|X_{\varepsilon}(t)-Z_{\varepsilon}(t)\right|^{2}>\delta_{4}\right)=0 .
$$

Proof. The proof is similar to Theorems 8 and 9.

Remark 11. By Theorems 8, 9, and 10, that is to say, we get the same results for three types of pathwise integrals of SDDEs.

\section{Examples}

Now we present two examples to demonstrate the procedure of the averaging principle. Define error $=X_{\varepsilon}(t)-Y_{\varepsilon}(t)$ in Figures 1 and 2 .

Example 12. Consider the following SDDE driven by fBm:

$$
\begin{aligned}
d X_{\varepsilon}(t)= & 2 a \varepsilon^{2 H} \sin ^{2} t X_{\varepsilon}(t) d t \\
& +c \varepsilon^{H} X_{\varepsilon}(t-1) d^{-} B^{H}(t), \quad t \geq 0,
\end{aligned}
$$

with an initial condition $X_{\varepsilon}(t)=t+1, t \in[-1,0]$, where $a, c$ are constants and $B^{H}(t)$ is a $\mathrm{fBm}$. Obviously,

$$
\begin{gathered}
b\left(t, X_{\varepsilon}(t)\right)=2 a \sin ^{2} t X_{\varepsilon}(t), \\
\sigma\left(t, X_{\varepsilon}(t-1)\right)=c X_{\varepsilon}(t-1) .
\end{gathered}
$$

Let

$$
\begin{aligned}
& \bar{b}\left(X_{\varepsilon}(t)\right)=\frac{1}{\pi} \int_{0}^{\pi} 2 a \sin ^{2} t X_{\varepsilon}(t) d t=a X_{\varepsilon}(t), \\
& \bar{\sigma}\left(X_{\varepsilon}(t)\right)=\frac{1}{\pi} \int_{0}^{\pi} c X_{\varepsilon}(t-1) d t=c X_{\varepsilon}(t-1) .
\end{aligned}
$$

And define an averaged SDDE as

$$
d Z_{\varepsilon}(t)=\varepsilon^{2 H} a Z_{\varepsilon}(t) d t+\varepsilon^{H} c Z_{\varepsilon}(t-1) d^{-} B^{H}(t) .
$$

On $t \in[0,1]$, the linear SDDE driven by fBm becomes linear SDE

$$
d Z_{\varepsilon}(t)=\varepsilon^{2 H} a Z_{\varepsilon}(t) d t+\varepsilon^{H} c t d^{-} B^{H}(t) .
$$

The explicit solution of this SDE is

$$
Z_{\varepsilon}(t)=\varepsilon^{2 H} \Phi(t) X(0)+\varepsilon^{H} c \Phi(t) \int_{0}^{t} s \Phi^{-1}(s) d B^{H}(s),
$$

where $\Phi^{-1}(t)=\exp (-a t)$

Repeating this procedure over the intervals $[1,2],[2,3]$, and so forth, we can obtain the explicit solution.
Because all the conditions $(\mathrm{C} 1)-(\mathrm{C} 7)$ are satisfied for function $b, \sigma, \bar{b}, \bar{\sigma}$ in SDDEs (19), (20), Theorems 8 and 9 hold. That is,

$$
E\left(\left|X_{\varepsilon}(t)-Z_{\varepsilon}(t)\right|^{2}\right) \leq \delta_{1}
$$

and as $\varepsilon \rightarrow 0$

$$
X_{\varepsilon}(t) \longrightarrow Z_{\varepsilon}(t) \quad \text { in probability. }
$$

Now we carry out the numerical simulation to get the solutions of (47) and (50) under the conditions of

(a) $X(0)=1, a=0.2, c=0.1, \varepsilon=0.045, H=0.55$,

(b) $X(0)=1, a=0.2, c=0.1, \varepsilon=0.045, H=0.65$,

(c) $X(0)=1, a=0.2, c=0.1, \varepsilon=0.045, H=0.75$,

(d) $X(0)=1, a=0.2, c=0.1, \varepsilon=0.045, H=0.85$, respectively.

Example 13. Consider the following SDDE driven by fBm:

$$
\begin{array}{rl}
d X_{\varepsilon}(t)= & a \varepsilon^{2 H} X_{\varepsilon}(t) d t \\
& +c \varepsilon^{H} \cos ^{2}\left(t+X_{\varepsilon}(t-1)\right) X_{\varepsilon}(t-1) d^{-} B^{H}(t), \\
t \geq 0 & t \geq 0
\end{array}
$$

with an initial condition $X_{\varepsilon}(t)=t+1, t \in[-1,0]$, where $a, c$ are constants and $B^{H}(t)$ is a $\mathrm{fBm}$. Obviously,

$$
\begin{gathered}
b\left(t, X_{\varepsilon}(t)\right)=a \varepsilon^{2 H} X_{\varepsilon}(t), \\
\sigma\left(t, X_{\varepsilon}(t-1)\right)=c \varepsilon^{H} \cos ^{2}\left(t+X_{\varepsilon}(t-1)\right) X_{\varepsilon}(t-1) .
\end{gathered}
$$

Let

$$
\begin{gathered}
\bar{b}\left(X_{\varepsilon}(t)\right)=\frac{1}{\pi} \int_{0}^{\pi} a \varepsilon^{2 H} X_{\varepsilon}(t) d t=a X_{\varepsilon}(t) \\
\bar{\sigma}\left(X_{\varepsilon}(t)\right)=\frac{1}{\pi} \int_{0}^{\pi} c \cos ^{2}\left(t+X_{\varepsilon}(t-1)\right) X_{\varepsilon}(t-1) d t \\
=\frac{c}{2} X_{\varepsilon}(t-1) .
\end{gathered}
$$

And define a new averaged equation as:

$$
d Z_{\varepsilon}(t)=\varepsilon^{2 H} a Z_{\varepsilon}(t) d t+\varepsilon^{H} \frac{c}{2} Z_{\varepsilon}(t-1) d^{-} B^{H}(t) .
$$

On $t \in[0,1]$, the SDDEs driven by fBm become a linear SDE

$$
d Z_{\varepsilon}(t)=\varepsilon^{2 H} a Z_{\varepsilon}(t) d t+\varepsilon^{H} \frac{c}{2} t d^{-} B^{H}(t)
$$




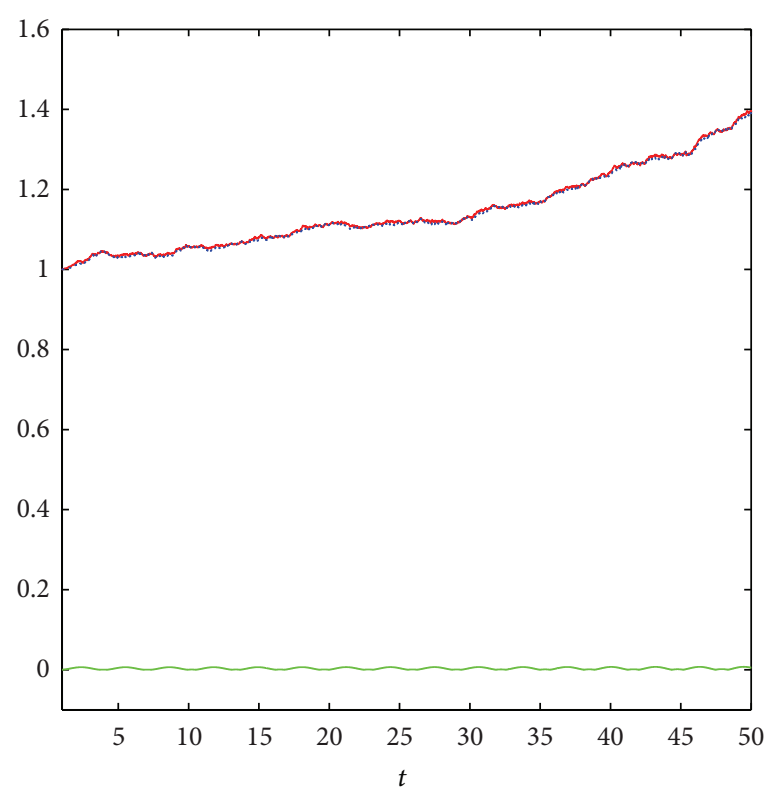

(a) $H=0.55$

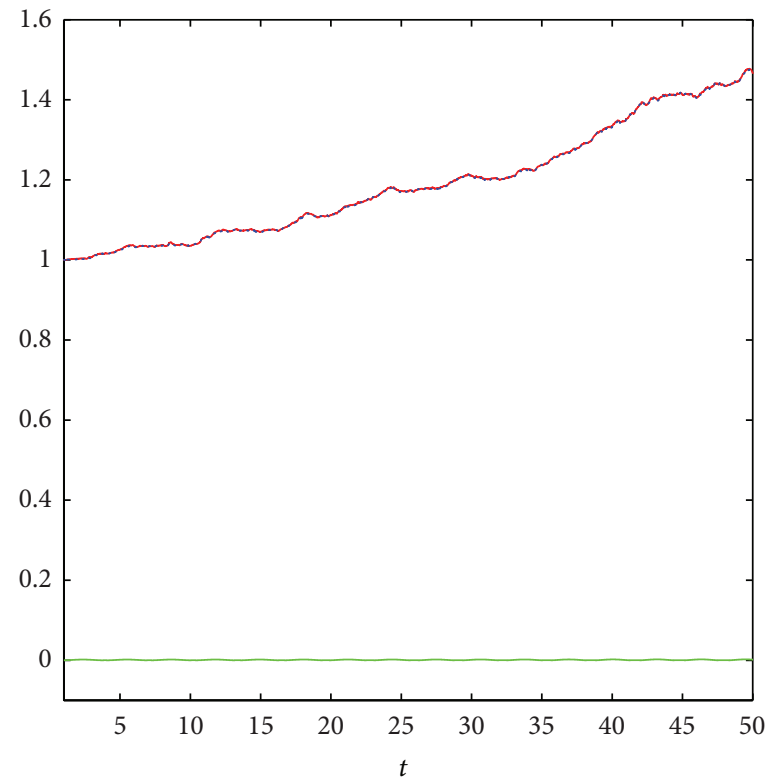

$X_{\varepsilon}(t)$
$\cdots . .$.
$Z_{\varepsilon}(t)$

Error

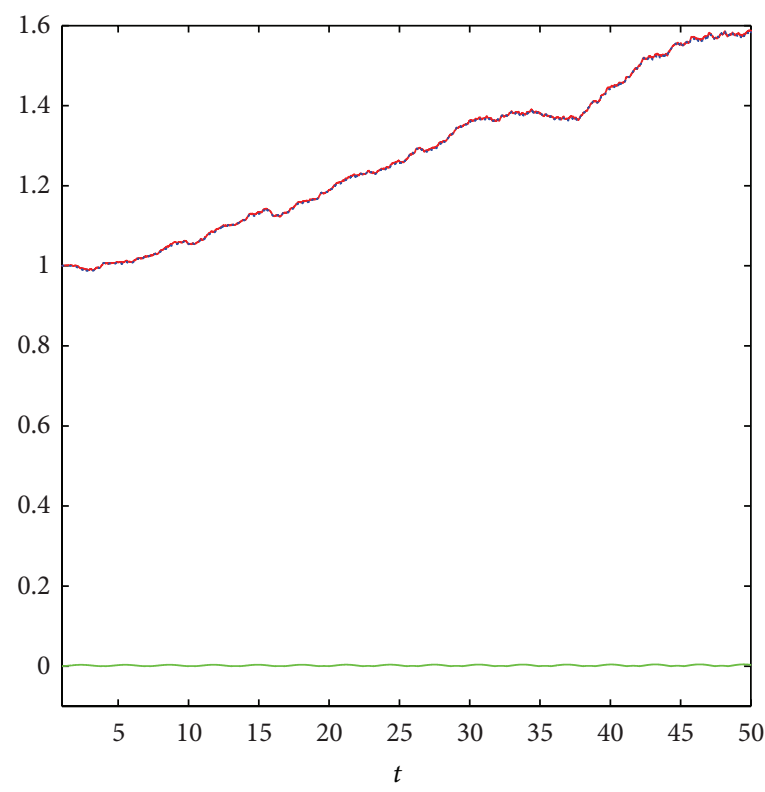

(b) $H=0.65$

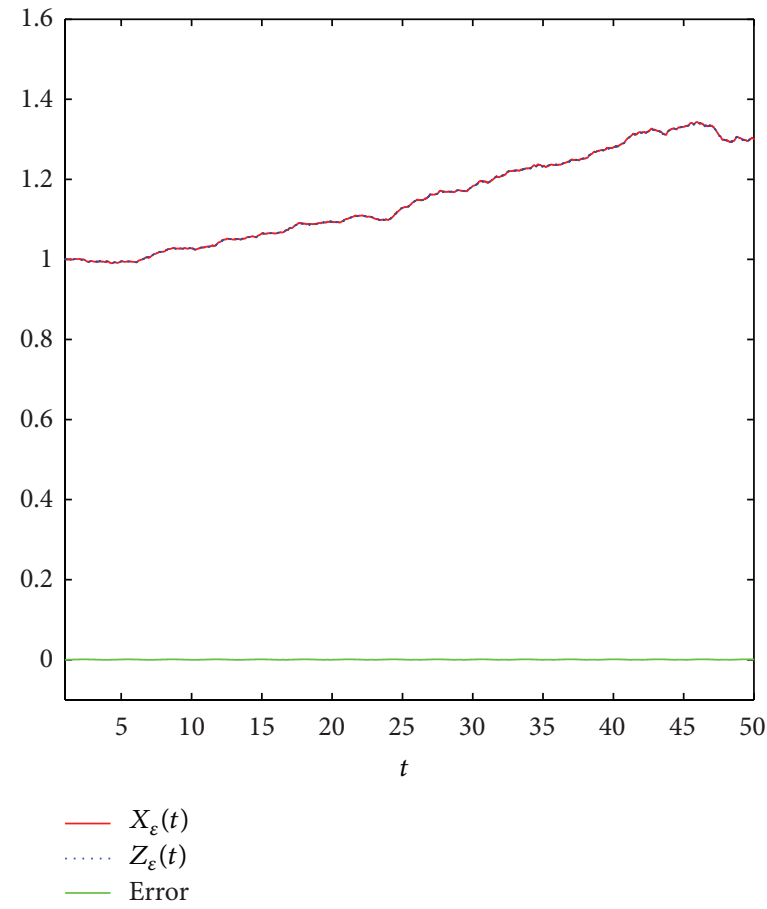

(d) $H=0.85$

FIGURE 1: Comparison of the exact solution $X_{\varepsilon}(t)$ with the averaged solution $Z_{\varepsilon}(t)$ for $(47)$ and (50) with $X(0)=1, a=0.2, c=0.1, \varepsilon=0.045$, and different $H$.

The explicit solution of this SDE is

$$
Z_{\varepsilon}(t)=\varepsilon^{2 H} \Phi(t) X(0)+\varepsilon^{H} \frac{c}{2} \Phi(t) \int_{0}^{t} s \Phi^{-1}(s) d^{-} B^{H}(s),
$$

where $\Phi^{-1}(t)=\exp (-a t)$
Repeating this procedure over the intervals $[1,2],[2,3]$, and so forth, we can obtain the explicit solution.

Because all the conditions (C1)-(C7) are satisfied for function $b, \sigma, \bar{b}, \bar{\sigma}$ in SDDEs (19) and (20), Theorems 8 and 9 hold. That is,

$$
E\left(\left|X_{\varepsilon}(t)-Z_{\varepsilon}(t)\right|^{2}\right) \leq \delta_{1}
$$




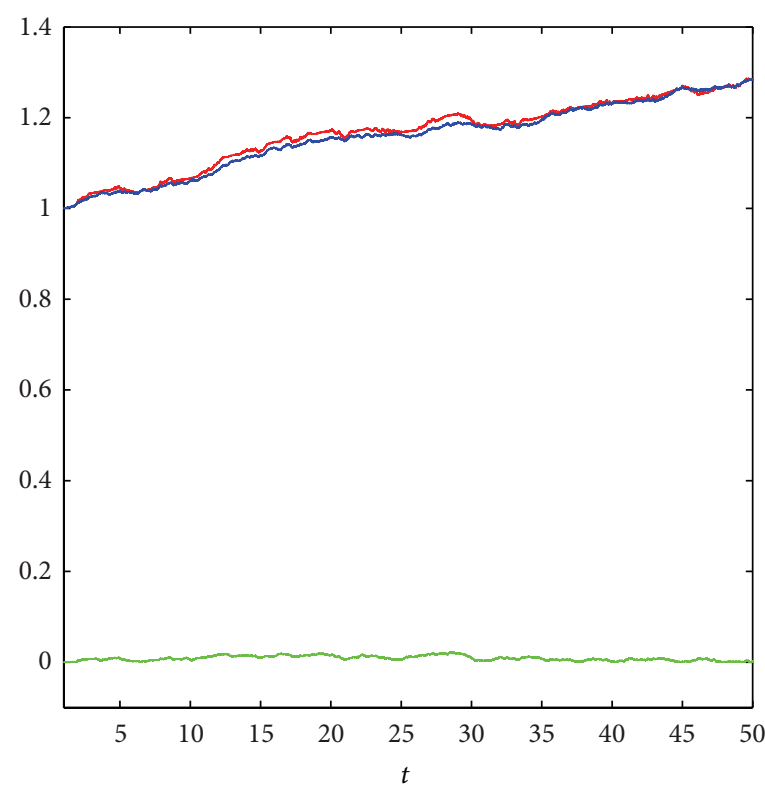

(a) $H=0.55$

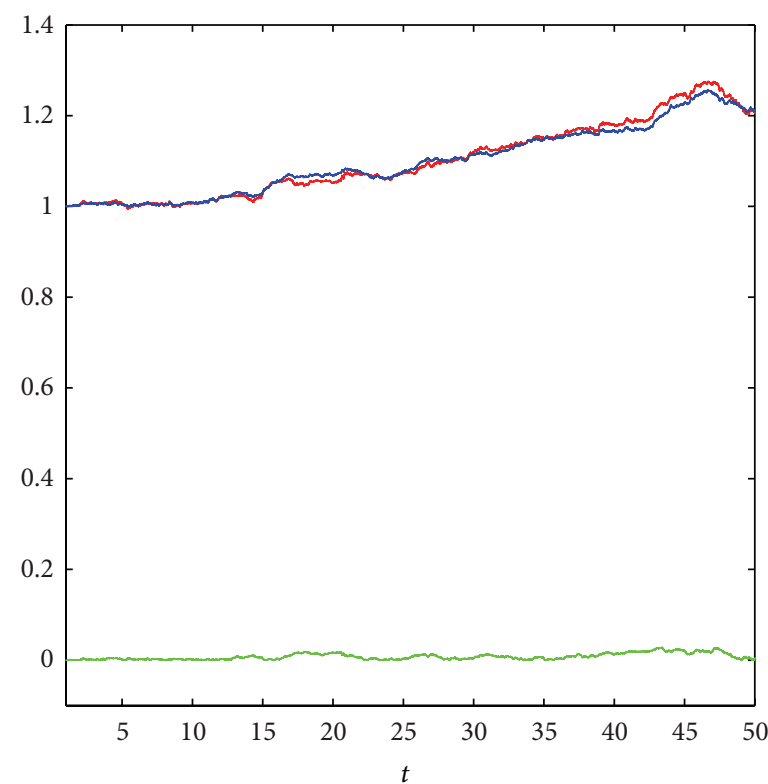

$X_{\varepsilon}(t)$
$-Z_{\varepsilon}(t)$
- Error

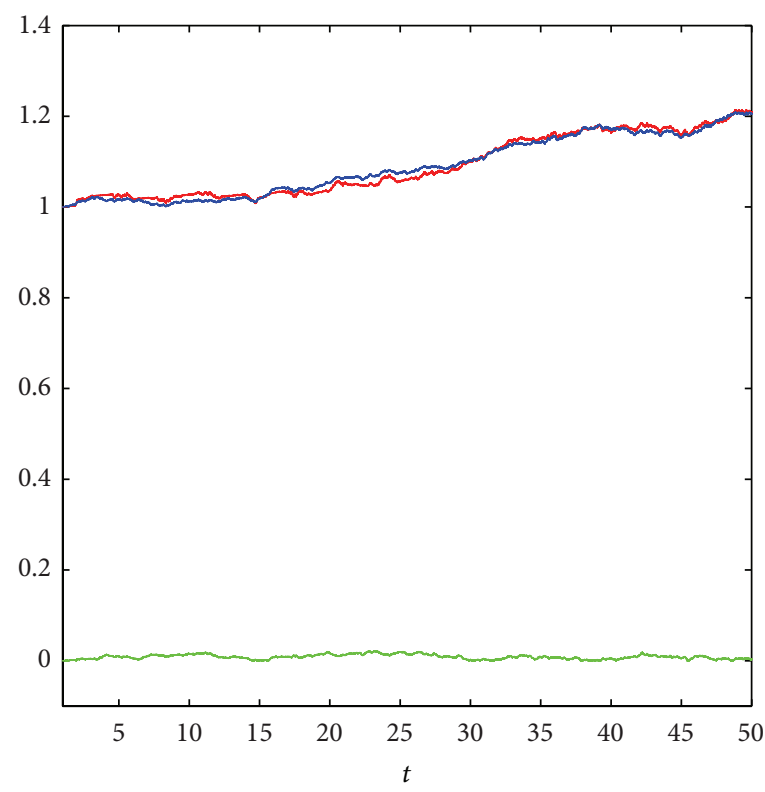

(b) $H=0.65$

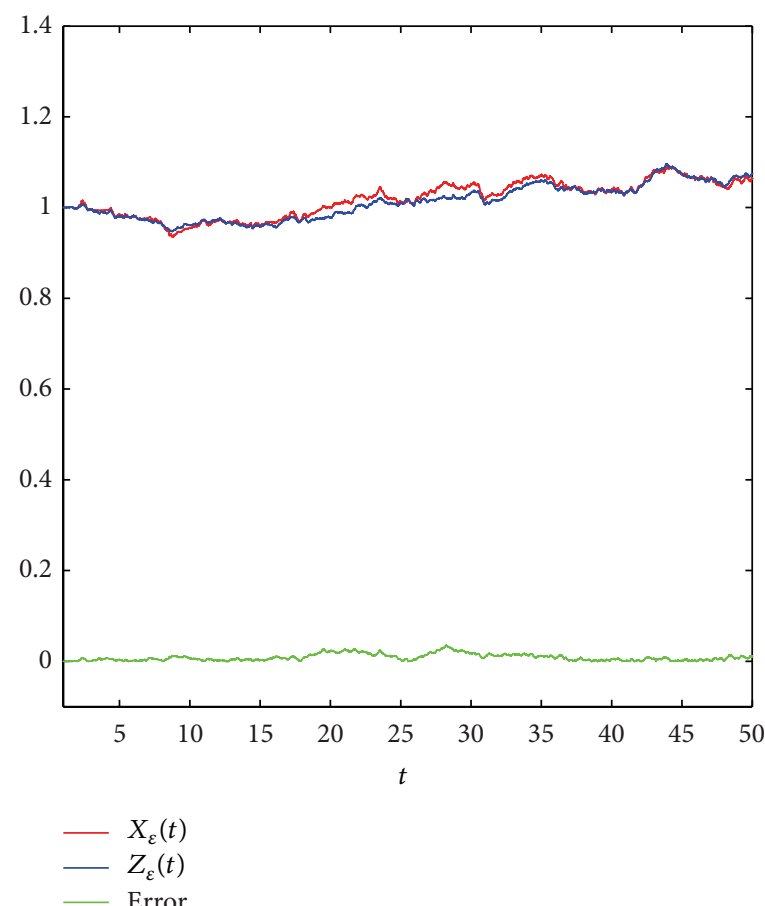

(d) $H=0.80$

(c) $H=0.70$

FIGURE 2: Comparison of the exact solution $X_{\varepsilon}(t)$ with the averaged solution $Z_{\varepsilon}(t)$ for (55) and (58) with $X(0)=1, a=0.2, c=0.01$, $\varepsilon=0.045$, and different $H$.

and as $\varepsilon \rightarrow 0$

$$
X_{\varepsilon}(t) \longrightarrow Z_{\varepsilon}(t) \quad \text { in probability. }
$$

Now we carry out the numerical simulation to get the solutions of (55) and (58) under the conditions of (a) $X(0)=1, a=0.2, c=0.01, \varepsilon=0.045, H=0.55$,

(b) $X(0)=1, a=0.2, c=0.01, \varepsilon=0.045, H=0.65$,

(c) $X(0)=1, a=0.2, c=0.01, \varepsilon=0.045, H=0.70$,

(d) $X(0)=1, a=0.2, c=0.01, \varepsilon=0.045, H=0.80$, respectively. 


\section{Conflict of Interests}

The authors declare that there is no conflict of interests regarding the publication of this paper.

\section{Acknowledgments}

This work was supported by the NSF of China (Grant no. 11372247) and Shaanxi Province, Program for NCET, SRF for ROCS, SEM, NPU Foundation for Fundamental Research and Graduate Starting Seed Fund.

\section{References}

[1] J. B. Roberts and P. D. Spanos, "Stochastic averaging: an approximate method of solving random vibration problems," International Journal of Non-Linear Mechanics, vol. 21, no. 2, pp. 111-134, 1986.

[2] W. Q. Zhu, "Recent developments and applications of the stochastic averaging method in random vibration," $A S M E$ Applied Mechanics Reviews, vol. 49, 10, pp. S72-S80, 1996.

[3] N. Sri Namachchivaya and Y. K. Lin, "Application of stochastic averaging for nonlinear dynamical systems with high damping," Probabilistic Engineering Mechanics, vol. 3, pp. 185-196, 1988.

[4] W. Q. Zhu, "Stochastic averaging methods in random vibration," ASME Applied Mechanics Reviews, vol. 41, no. 5, pp. 189-199, 1988.

[5] R. L. Stratonovich, Topics in the Theory of Random Noise, Gordon and Breach, New York, NY, USA, 1967.

[6] R. L. Stratonovich, Conditional Markov Processes and their Application to the Theory of Optimal Control, Elsevier, New York, NY, USA, 1967.

[7] R. Z. Khasminskii, "A limit theorem for the solution of differential equations with random right-hand sides," Theory of Probability \& Its Applications, vol. 11, pp. 390-405, 1963.

[8] R. Z. Khasminskii, "Principle of averaging of parabolic and elliptic differential equations for Markov process with small diffusion," Theory of Probability \& Its Applications, vol. 8, no. 1, pp. 1-21, 1963.

[9] I. M. Stoyanov and D. D. Bainov, "The averaging method for a class of stochastic differential equations," Ukrainian Mathematical Journal, vol. 26, no. 2, pp. 186-194, 1974.

[10] V. G. Kolomiets and A. I. Mel'nikov, "Averaging of stochastic systems of integral-differential equations with Poisson noise," Ukrainian Mathematical Journal, vol. 43, no. 2, pp. 242-246, 1991.

[11] W. Q. Zhu, "Nonlinear stochastic dynamics and control in Hamiltonian formulation," ASME Applied Mechanics Reviews, vol. 59, no. 4, pp. 230-248, 2006.

[12] W. T. Jia, W. Q. Zhu, and Y. Xu, "Stochastic averaging of quasinon-integrable Hamiltonian systems under combined Gaussian and Poisson white noise excitations," International Journal of Non-Linear Mechanics, vol. 51, pp. 45-53, 2012.

[13] Y. Zeng and W. Q. Zhu, "Stochastic averaging of quasinonintegrable-Hamiltonian systems under Poisson white noise excitation," ASME Journal of Applied Mechanics, vol. 78, no. 2, Article ID 021002, 11 pages, 2011.

[14] Y. Xu, J. Duan, and W. Xu, "An averaging principle for stochastic dynamical systems with Lévy noise," Physica D, vol. 240, no. 17, pp. 1395-1401, 2011.
[15] W. E. Leland, M. S. Taqqu, W. Willinger, and D. V. Wilson, "On the self-similar nature of Ethernet traffic (extended version)," IEEE/ACM Transactions on Networking, vol. 2, no. 1, pp. 1-15, 1994.

[16] Y. Hu and B. Øksendal, "Fractional white noise calculus and applications to finance," Infinite Dimensional Analysis, Quantum Probability and Related Topics, vol. 6, no. 1, pp. 1-32, 2003.

[17] R. Scheffer and F. R. Maciel, "The fractional Brownian motion as a model for an industrial airlift reactor," Chemical Engineering Science, vol. 56, no. 2, pp. 707-711, 2001.

[18] N. Chakravarti and K. L. Sebastian, "Fractional Brownian motion models for polymers," Chemical Physics Letters, vol. 267, no. 1-2, pp. 9-13, 1997.

[19] Y. Xu and R. Guo, "Stochastic averaging principle for dynamical systems with fractional brownian motion," AIMS Discrete and Continuous Dynamical Systems B. In press.

[20] T. Taniguchi, K. Liu, and A. Truman, "Existence, uniqueness, and asymptotic behavior of mild solutions to stochastic functional differential equations in Hilbert spaces," Journal of Differential Equations, vol. 181, no. 1, pp. 72-91, 2002.

[21] X. Mao, "Numerical solutions of stochastic functional differential equations," LMS Journal of Computation and Mathematics, vol. 6, pp. 141-161, 2003.

[22] L. Tan and D. Lei, "The averaging method for stochastic differential delay equations under non-Lipschitz conditions," Advances in Difference Equations, vol. 2013, article 38, 2013.

[23] F. Biagini, Y. Hu, B. Øksendal, and T. Zhang, Stochastic Calculus for Fractional Brownian Motion and Applications, Springer, London, UK, 2008.

[24] T. E. Duncan, Y. Hu, and B. Pasik-Duncan, "Stochastic calculus for fractional Brownian motion I. Theory," SIAM Journal on Control and Optimization, vol. 38, no. 2, pp. 582-612, 2000.

[25] Y. S. Mishura, Stochastic Calculus for Fractional Brownian Motion and Related Processes, vol. 1929 of Lecture Notes in Mathematics, Springer, Berlin, Germany, 2008.

[26] E. Alòs and D. Nualart, "Stochastic integration with respect to the fractional Brownian motion," Stochastics and Stochastics Reports, vol. 75, no. 3, pp. 129-152, 2003.

[27] F. Russo and P. Vallois, "Forward, backward and symmetric stochastic integration," Probability Theory and Related Fields, vol. 97, no. 3, pp. 403-421, 1993.

[28] L. C. Young, "An inequality of the Hölder type, connected with Stieltjes integration," Acta Mathematica, vol. 67, no. 1, pp. 251282,1936

[29] D. Nualart and G. Via, "Stochastic integration with respect to fractional Brownian motion and applications," Contemporary Mathematics, vol. 336, pp. 3-39, 2003.

[30] M. Ferrante and C. Rovira, "Convergence of delay differential equations driven by fractional Brownian motion," Journal of Evolution Equations, vol. 10, no. 4, pp. 761-783, 2010. 


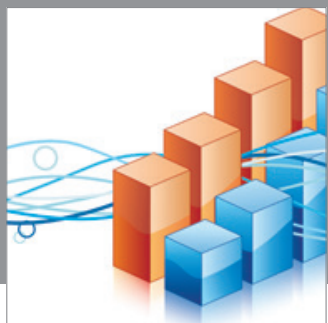

Advances in

Operations Research

mansans

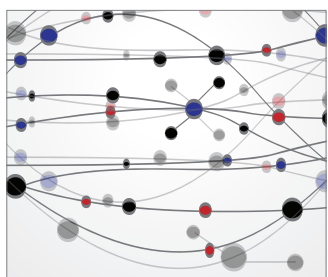

The Scientific World Journal
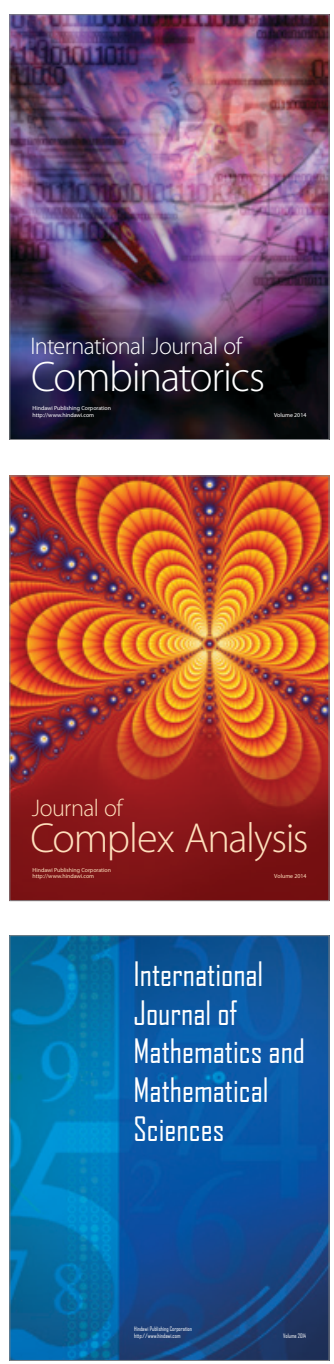
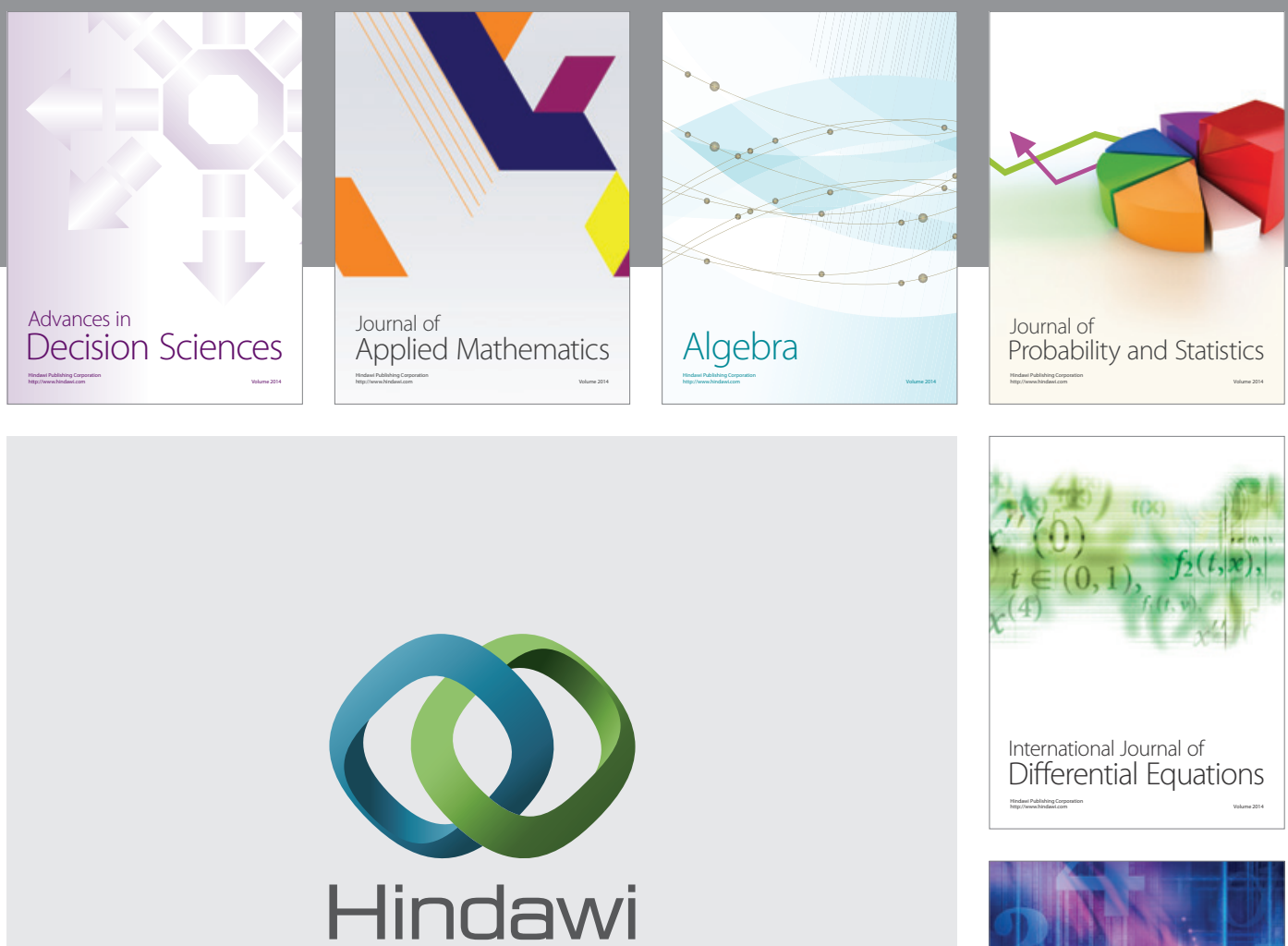

Submit your manuscripts at http://www.hindawi.com
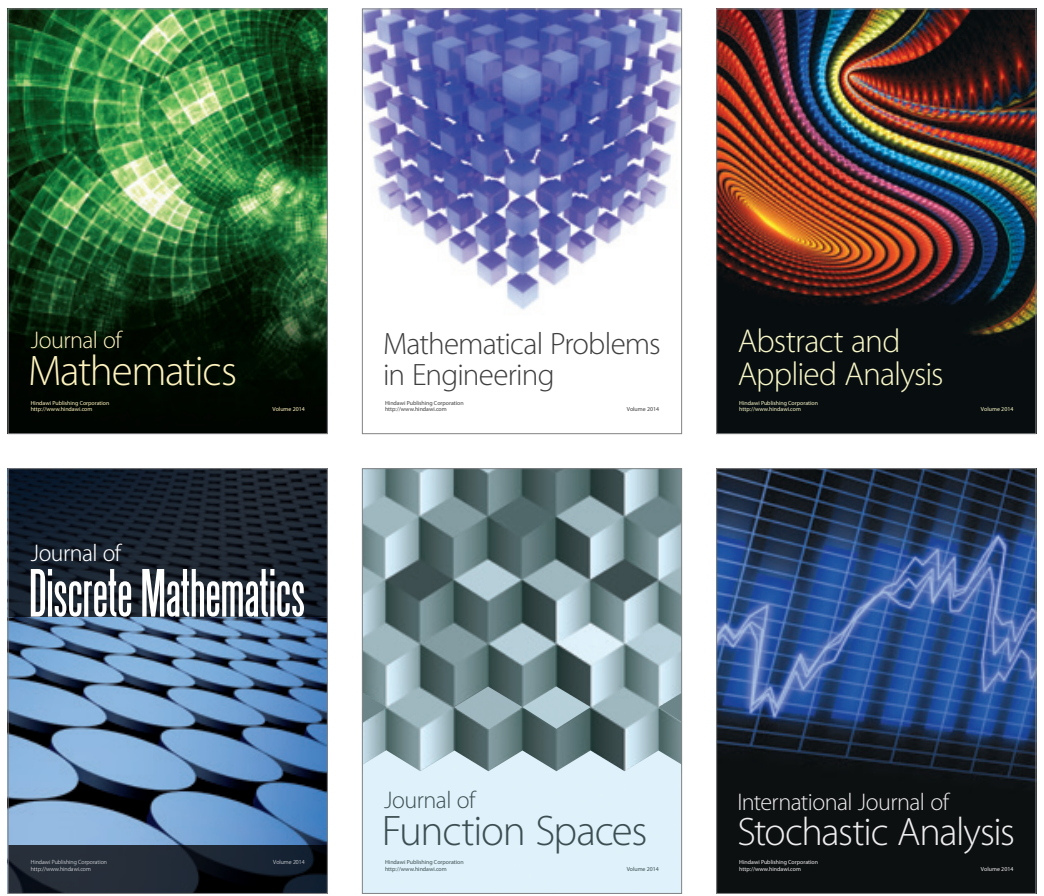

Journal of

Function Spaces

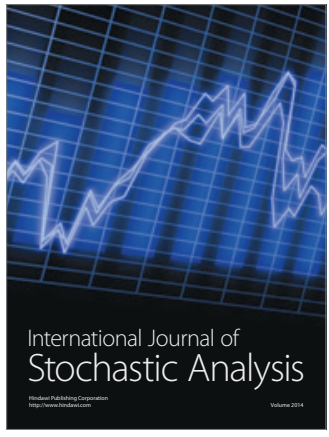

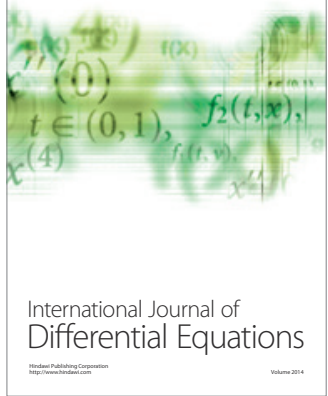
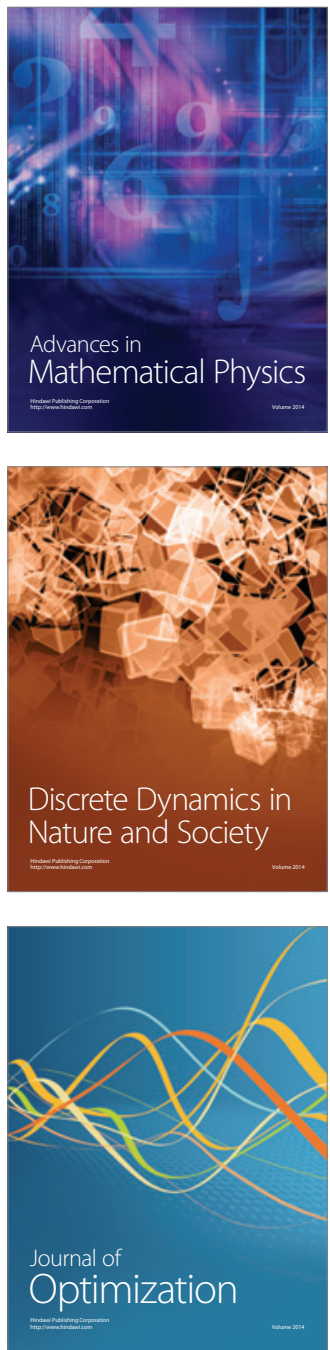